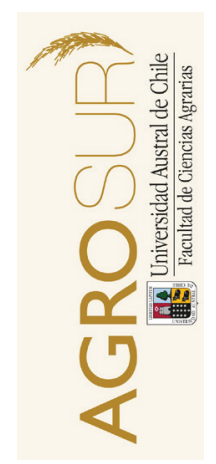

\title{
Influencia de la adición de humo líquido en la estabilidad y aceptabilidad de chorizo ahumado
}

\author{
Influence of adding liquid smoke on stability and acceptability \\ of smoked sausage
}

\author{
Maldonado Chávez, A.P. ${ }^{a *}$, Mira Vásquez, J.M. ${ }^{b}$, Pólit Corral, P. ${ }^{a}$ \\ ${ }^{a}$ Escuela Politécnica Nacional, Facultad de Ingeniería Química y Agroindustria, \\ Ladrón de Guevara E11-254. Quito, Ecuador. \\ ${ }^{b}$ Escuela Superior Politécnica de Chimborazo, Facultad de Ingeniería en Industrias Pecuarias, \\ Panamericana Sur km 1 11/2, Riobamba, Ecuador.
}

\begin{tabular}{l} 
A R T I C L E I N F O \\
\hline Keywords: \\
Liquid smoked sausage \\
Shelf-life \\
Sensorial analysis \\
Microbiological stability \\
Food preservative \\
\hline Original Research Article, \\
Food Science \\
\hline *Corresponding author: \\
Paola Maldonado \\
E-mail address: \\
paolamaldonado2485@ \\
hotmail.com
\end{tabular}

hotmail.com

\begin{abstract}
A B S T R A C T
This research is about the influence of liquid smoke on the stability and acceptability of smoked sausage. The product was made based on an ingredient formula. Three levels of liquid smoke $(0.3 ; 0.6$ and $0.9 \%)$ along with a control treatment $(0.0 \%)$ were studied. Four replications for each treatment were performed. The finished product was stored under refrigeration at $4{ }^{\circ} \mathrm{C}$ in plastic trays wrapped with polyethylene film. Product characterization included the content of protein, fat, moisture and ash. Also, an acceptability study was performed through sensory analysis of the following organoleptic parameters: flavor, aroma, color, texture and appearance. Subsequently, a stability study was conducted at ambient conditions $\left(4{ }^{\circ} \mathrm{C}, 60 \% \mathrm{RH}\right)$, evaluating microbiological parameters (Escherichia coli, total coliform and fungi) as well as the mentioned organoleptic parameters. Shelf life was determined for each of the treatments. These analyzes were performed on days $0,10,20$ and 30. For analysis of the results, statistical tests were conducted in order to determine the existence of statistically significant differences between treatments. Finally, it was possible to demonstrate the efficacy of liquid smoke as a food preservative, with increasing concentration of liquid smoke, a significant decrease in microbial contamination occurred. Moreover, treatment with $0.6 \%$ liquid smoke had the most stable organoleptic parameters, with a product's shelf life of 43 days.
\end{abstract}

\section{RESUMEN}

La presente investigación es acerca de la influencia de humo líquido, en la estabilidad y aceptabilidad del chorizo ahumado. El producto se trabajó en base a una fórmula establecida de los ingredientes y con tres niveles de humo líquido 0,3, 0,6 y $0,9 \%$ además de un tratamiento control $(0,0 \%)$. Se realizaron cuatro repeticiones del proceso para cada tratamiento. El producto elaborado se almacenó bajo refrigeración a $4{ }^{\circ} \mathrm{C}$ en bandejas plásticas cubierto de lámina de polietileno. Se realizó la caracterización del producto, determinando el contenido de proteína, grasa, humedad y cenizas. También se realizó un estudio de aceptabilidad, donde se evaluó los siguientes parámetros organolépticos: sabor, aroma, color, textura y apariencia. Posteriormente, se realizó un estudio de estabilidad en condiciones ambientales $\left(4{ }^{\circ} \mathrm{C}, 60 \% \mathrm{HR}\right)$, donde se evaluaron los parámetros microbiológicos (Escherichia coli, coliformes totales y hongos), además de los parámetros organolépticos mencionados y se determinó el tiempo de vida útil de cada uno de los tratamientos. Estos análisis se realizaron los días: 0,10, 20 y 30. Para el análisis de resultados, se realizó un estudio estadístico, el cual permitió establecer la existencia o no de diferencias estadísticas significativas entre los tratamientos. Finalmente, se determinó que el humo líquido es un buen conservante de los alimentos, ya que a mayor concentración del mismo en el producto fue menor la contaminación microbiana. Además, el tratamiento con 0,6\% de humo líquido fue el más estable organolépticamente, con una vida útil de 43 días.

Palabras clave: chorizo ahumado con humo líquido, vida útil, análisis sensorial, estabilidad microbiológica, conservación de alimentos.

\section{INTRODUCCIÓN}

Históricamente, la carne ha sido uno de los principales alimentos que ha consumido el hombre. Actualmente, debido a sus bondades nutritivas y al desarrollo de la industria alimenticia, ha permitido que la carne sea uno de los alimentos más consumidos (Ranken, 2003). En Ecuador durante los últimos años se aprecia un aumento en la demanda de embutidos, que se podría explicar por el aumento en el consumo de comida rápida y pre cocida, que antes no era común en Ecuador, el poco tiempo que disponen las mujeres que com- 
binan el trabajo con las tareas del hogar, el aumento en el consumo de embutidos con bajo contenido de grasa y el aumento en el consumo de embutidos Gourmet. En Ecuador se producen entre 36 y 50 millones de kilogramos de embutidos anualmente; es decir que cada ecuatoriano consume entre 2,77 y 3,85 kg por año. De todos los embutidos existentes, los más apetecidos son las mortadelas y las salchichas, que juntas representan el $75 \%$ de la producción nacional. Le siguen los chorizos con $14 \%$, jamones con $5 \%$ y el $6 \%$ restante pertenece a otras presentaciones (Barzola, 2013).

El chorizo es el embutido elaborado con carne de animales de abasto, solas o en mezcla, con ingredientes y aditivos de uso permitido y embutidos en tripas naturales o artificiales de uso permitido; puede ser fresco o crudo, cocido, madurado, ahumado o sin ahumado (INEN 2012; Essien, 2003). El proceso tradicional de fabricación del chorizo típico incluye las siguientes fases: picado, mezclado, embutido, atado, cocción, ahumado, maduración y empacado (Gracey, 2001). Durante el tiempo de maduración existen procesos de desecación y adquisición de firmeza en la textura, a la vez que se desarrolla el aroma, fruto de la suma de los aromas naturales y los resultantes de la actividad microbiana sobre los componentes de la masa del embutido (Barco, 2008).

Se han desarrollado ciertas técnicas de conservación de los alimentos como el ahumado. Su consumo se remonta a épocas prehistóricas. Las propiedades inhibidoras del humo son bastamente reconocidas desde entonces, y han sido objeto de innumerables estudios a través de la historia (Cid, 2004). Con la llegada del humo líquido preparado a partir de madera, desde fines del siglo 19, se estableció una forma más práctica y versátil de aplicación del humo tradicional. Hoy su uso está bastante difundido en el mercado (Restrepo, 2012). Entre todos los componentes naturales en el humo, la presencia de fenoles, carbonilos y ácidos orgánicos hace posible el efecto antioxidante, bactericida y bacteriostático del humo líquido (Yusnaini et al., 2012; Girard, 2000). En general, el ahumado introduce en los alimentos altos en proteínas sus componentes aromáticos que imparten sabor y color característico a este tipo de productos (Amran y Abbas, 2011). Además brinda otras ventajas para la industria alimenticia respecto al ahumado tradicional como: uniformidad de sabor y color de los alimentos, producción más limpia, control de emisiones por gases y remoción de sustancias peligrosas (Pico, 2008). El presente trabajo estudia la influencia de la adición del humo líquido en la estabilidad y en la aceptabilidad de chorizo especial ahumado con características organolépticas, bromatológicas y microbiológicas requeridas en el mercado ecuatoriano y se determinó la vida útil con respecto a la concentración de humo líquido. Con esto se pretende dar a la industria cárnica una alternativa en el proceso de fabricación de este tipo de embutidos.

\section{MATERIALES Y MÉTODOS}

\section{Materiales}

Para la elaboración del chorizo ahumado se utilizó carne de cerdo (pierna), carne de vacuno (pulpa redonda), grasa de cerdo, conservantes (sal común, nitrito de sodio, tripolifosfato de sodio, eritorbato de sodio, humo líquido), condimentos (pimienta negra, ajo en polvo, comino, orégano en polvo, condimento de chorizo, achiote en polvo), tripa de cerdo. La unidad experimental constó de $3 \mathrm{~kg}$ de producto. Los conservantes usados son de marca Alitecno (Perú).

\section{Métodos}

\section{Elaboración del producto}

Para la elaboración del producto, se realizó el proceso que se describe a continuación: deshuesado y picado para separar la carne pura del hueso, se eliminaron partes de la carne que no son usadas en el proceso y se picó la carne en trozos medianos; para el molido de la carne y de la grasa de cerdo se utilizó un molino de disco de 8 mm (TCN-32, Iozzelli, Italia) con una capacidad de 100 $\mathrm{kg} / \mathrm{h}$; la cantidad de carne, grasa de cerdo, aditivos y condimentos para cada uno de los tratamientos se midió con una balanza de precisión $( \pm 0,1 \mathrm{~g}$ ) (PCE Group, BSH 6000, España); se mezcló manualmente las carnes con la grasa de cerdo, luego se añadió los aditivos, condimentos y la cantidad correspondiente de humo líquido; el tiempo de mezclado fue de aproximadamente 20 minutos por tratamiento; para el embutido de la masa de cada uno de los tratamientos en la tripa natural se utilizó una máquina embutidora hidráulica (capacidad $30 \mathrm{~kg} / \mathrm{h}$; INV 25, Iozzelli, , Italia); el atado del producto final fue en porciones de $12 \mathrm{~cm}$, el horneado fue por 30 minutos hasta que el producto alcanzó una temperatura interna de $68^{\circ} \mathrm{C}$ en un horno ahumador de armario (capacidad 100 kg; GG-430, Cherri Gino, Italia); el producto fue enfriado a temperatura ambiente por una hora y almacenado a una temperatura de $4{ }^{\circ} \mathrm{C}$ en bandejas de plástico cubiertas con lámina de polietileno.

\section{Caracterización bromatológica y microbiológica del producto}

Para las mediciones experimentales se tomaron muestras de $200 \mathrm{~g}$ por cada tratamiento y se enviaron al laboratorio donde se realizaron las siguientes mediciones: caracterización bromatológica y microbiológica. En la caracterización bromatológica se determinó el contenido de humedad (método AOAC 937.09 volumétrico), grasa, proteína y cenizas (método AOAC 923.03 gravimétrico); para el análisis microbiológico del producto las mediciones experimentales consideradas 
fueron: recuento de Escherichia coli, (UFC/g), coliformes totales (UFC/g) mediante la técnica de siembra en profundidad y recuento de hongos (UFC/g) mediante la técnica de siembra en extensión.

\section{Caracterización sensorial del producto}

Para el estudio de aceptación del producto se evaluaron los siguientes cinco parámetros sensoriales: apariencia, color, sabor, aroma, y textura. Se evaluó a través de un análisis sensorial usando la Escala Hedónica con pautas no estructuradas de 0 a 15 centímetros, en donde se miden las condiciones psicológicas de agrado, desagrado o la indiferencia de cada una de las muestras de producto a evaluar (Araya, 2003; Sancho, 2002), asignando luego un puntaje máximo de 5 puntos. El ensayo se llevó a cabo en el Centro de Producción de Cárnicos de la Escuela Superior Politécnica de Chimborazo, con un panel conformado por 15 personas, entre 20 y 65 años. Se presentaron las muestras de chorizos sobre bandejas. Para evaluar cada una de las fórmulas, se seleccionó una distribución al azar de los tratamientos Para cada prueba los panelistas evaluaron en un formulario las 5 características sensoriales. Para neutralizar el sabor entre pruebas se proporcionó galletas de soda y agua pura a los panelistas. El estudio fue realizado en 3 repeticiones.

\section{Análisis de Estabilidad del Producto.}

Para el análisis de estabilidad se almacenó el chorizo en bandejas plásticas cubiertas de láminas de polietileno. Las condiciones de almacenamiento fueron a una temperatura de $4{ }^{\circ} \mathrm{C}$, una humedad relativa de $60 \%$, los tiempos de medición para el análisis fueron: 0 , 10,20 y 30 días. Los parámetros de medición microbiológicos fueron recuentos de Escherichia coli, coliformes totales y hongos. Sin embargo, por considerarse que los hongos no son un factor determinante en la estabilidad del producto, según las normas Oficiales del Codex Alimentarius, CODEX STAN 98-1981 (Rev. 3. - 2015) y la norma INEN NTE 1338 para productos cárnicos, este factor fue tomado en cuenta sólo para comprobar la acción antimicrobiana del humo líquido. Los parámetros organolépticos fueron los mismos que se determinaron para el estudio de aceptabilidad (apariencia, color, sabor, aroma, y textura) mediante el método de comparación múltiple de diferencia. Para el cálculo del tiempo de vida útil del chorizo ahumado, para cada uno de los tratamientos, se aplicó la fórmula de cinética de orden cero de acuerdo a la ecuación 1.

$$
A_{f}=A_{0}+k t
$$

Donde: $A_{f}$ es la concentración final del factor al tiempo, $A_{0}$ es la concentración inicial del factor al tiempo $0, t$ es el tiempo de estabilidad (días) y $k$ es la constante cinética a la temperatura estudiada (Taoukis y Labuza, 1989).

Se consideraron como valores críticos, aquellos que indican que la calidad del producto no es aceptable. Estos son los siguientes: coliformes totales $<10 \mathrm{UFC} / \mathrm{g}$ (INEN, 2012), apariencia, sabor y aroma, color y textura < 3. Para el cálculo de la vida útil del producto, se trabajó con los valores promedios obtenidos luego del análisis estadístico; con los mismos, se graficó y se obtuvo la recta de comportamiento de la propiedad evaluada en el tiempo con las respectivas pendientes y coeficiente de determinación $\mathrm{R}^{2}$, para cada uno de los tratamientos. Para los resultados de las mediciones organolépticas, primero se realizó una ponderación acorde a la siguiente escala: apariencia (30\%), sabor y aroma (30\%), color (25\%) y textura (15\%). De este estudio se realizaron 3 repeticiones para cada uno de los tratamientos.

\section{Análisis estadísticos}

Con los datos obtenidos de la prueba organoléptica, se aplicó un diseño completamente al azar. Los datos se procesaron con el programa estadístico SAS® (Statistical Analysis System, versión 9.1. SAS Institute Inc). Se realizaron los siguientes análisis: análisis de varianza para las diferencias, separación de medias según Tukey al 5\%, coeficiente de variación (\%). Para el estudio se trabajó con tres tratamientos, en los cuales variaba la concentración de humo líquido dentro del chorizo $(0,3$; $0,6$ y $0,9 \%)$, correspondientes al T1, T2 y T3 más el testigo o tratamiento control $(0,0 \%)$ que se denomina $\mathrm{T} 0$, con cuatro repeticiones por cada tratamiento para cada uno de los ensayos realizados.

\section{RESULTADOS Y DISCUSIÓN}

\section{Caracterización del Producto Terminado}

En el Cuadro 1, se puede observar el análisis proximal del chorizo ahumado con humo líquido de cada uno de los tratamientos aplicados. Los distintos tratamientos presentan contenidos de 21,39; 20,95; 21,06 y $21,06 \%$ de proteína, entre los cuales, como se esperaba, no se registraban diferencias estadísticas significativas, los mismos son superiores a los mínimos reportados por la norma INEN NTE 1338, que manifiesta que, el chorizo maduro debe tener $14 \%$ de proteína, y el chorizo crudo y escaldado $12 \%$, según el método de ensayo INEN NTE 781. Lo mismo sucede con los contenidos de grasa y cenizas. Estos valores y en especial el de proteína se cree que se debe a la calidad de las materias primas utilizadas. También la disminución de humedad dentro del producto como resultado de la cocción hace 
Cuadro 1. Características físico químicas del chorizo como respuesta a la aplicación de humo líquido.

Table 1. Physico-chemical characteristics of sausage after application of liquid smoke.

\begin{tabular}{ccccc}
\hline \multirow{2}{*}{ VARIABLES } & \multicolumn{4}{c}{ PORCENTAJE DE HUMO LÍQUIDO (\%) } \\
\cline { 2 - 5 } & $\mathbf{0 , 0 0}$ & $\mathbf{0 , 3 0}$ & $\mathbf{0 , 6 0}$ & $\mathbf{0 , 9 0}$ \\
\hline Proteína & 21,39 & 20,95 & 21,06 & 21,06 \\
Grasa & 24,17 & 22,73 & 24,86 & 22,79 \\
Humedad & 49,66 & 50,91 & 50,47 & 51,13 \\
Cenizas & 3,47 & 3,78 & 3,43 & 3,77 \\
\hline
\end{tabular}

que los valores de proteína, grasas y ceniza aumente (Alcicek, 2014).

Respecto al perfil microbiológico, el producto no presenta coliformes totales, lo que demuestra que no existió contaminación post-tratamiento térmico (Anmat, 2003); además que la cocción del producto fue la adecuada $\left(68{ }^{\circ} \mathrm{C}\right.$ temperatura interna) como lo recomienda Mira (1998) para la elaboración de este tipo de embutidos. Además no existió contaminación de Escherichia coli, lo que garantizó la ausencia de materia fecal en la materia prima (Jay, 2002). Sin embargo, hubo presencia de hongos en todos los tratamientos desde 45 UFC/g para el T0; 20, 15 y 10 UFC/g para T1, T2 y T3 respectivamente, existiendo diferencias estadísticamente significativas $(p<0,05)$ con el tratamiento control; con estos resultados se puede observar la acción positiva del humo líquido, que indica que a mayor cantidad del mismo en el chorizo ahumado, la cantidad de hongos es menor, debido a las características antimicrobianas que posee el humo líquido por el contenido de compuestos fenólicos, carbonilos y ácidos orgánicos (Milly et al., 2005). Como se esperaba, el humo líquido retrasó el crecimiento microbiológico en el producto.

La aceptabilidad del producto se evaluó a través del método de la Escala Hedónica con pautas no estructuradas para medir el agrado, desagrado o la indiferencia hacia el producto. La aceptabilidad del producto se vio influenciada por una serie de factores entre los cuales están los factores fisiológicos internos que regulan el hambre (Cordero, 2013), por ello se escogió el horario del estudio de aceptabilidad y evaluación organoléptica alrededor de las 11:30 y 12:00 h, ya que a esa hora del día los panelistas están con un estado de apetito normal y tampoco llenos porque ya había pasado algunas horas desde el desayuno, por lo que se encontraban dispuestos a realizar la degustación del producto.

El sabor fue una parte muy importante sobre todas las sensaciones que son percibidas durante la degustación del producto, ya que esta sensación es una estimulación simultánea de sensaciones químicas como el gusto y el olor por un complejo mixto de moléculas densas y volátiles (Ohloff et al., 1985). La textura, es otra característica organoléptica que tuvo un importante efecto de aceptación del producto y que en algunas ocasiones fue mucho más importante que el sabor, ya que mediante este atributo se pudo evaluar otros parámetros tal como verificar si el tiempo de mezcla y la cantidad de tripolifosfato fueron los adecuados, ya que estos permiten la emulsión de las proteínas para formar la masa, la misma que no debe deshacerse con facilidad (Pico, 2008). La apariencia también jugó un papel importante en la aceptación y percepción del producto ya que la psicología también interviene en los estímulos visuales (Moswittz, 1983). A escala general, todos los tratamientos estudiados son aceptados y no se observa diferencia estadística significativa $(p>0,05)$. Entre los tratamientos realizados, los resultados fueron $4,40 \pm 0,50,4,60 \pm 0,50,4,80 \pm 0,50$ y $4,20 \pm 0,50$ sobre una escala de 5,00. Sin embargo, se observa que el T2 es el tratamiento de mayor aceptación (92,70\%), y el T3 él de menor aceptación $(86,45 \%)$, esto puede ser el resultado de que el sabor a humo líquido es más fuerte en esta concentración dentro del producto. En el estudio realizado por Özpolat (2015) se determinó que el humo líquido es más perceptible sensorialmente que un producto ahumado de forma tradicional y más aún cuando la concentración de éste aumenta.

\section{Estudio de Estabilidad}

\section{Características Organolépticas del Producto}

En el Cuadro 2 se puede observar el desarrollo del perfil sensorial del producto durante el período de almacenaje. La apariencia del chorizo tuvo una calificación muy buena en todos los tratamientos y no se observaron diferencias estadísticamente significativas ( $\mathrm{p}<0,05)$; a los 10 días de almacenamiento sobre todo en los tratamientos T0, T1 y T2 un leve mejoramiento de la apariencia fue percibido por los jueces, lo que se mantuvo hasta el día 20, para recaer al día 30, en todos los tratamientos. Esto se debió al cambio del producto 
Cuadro 2. Evolución de las características organolépticas del chorizo durante el almacenado.

Table 2. Characteristics of sausage during storage time.

\begin{tabular}{|c|c|c|c|c|c|c|c|c|c|c|}
\hline \multirow{2}{*}{ VARIABLE } & \multirow{2}{*}{$\begin{array}{l}\text { TIEMPO } \\
\text { (días) }\end{array}$} & \multicolumn{8}{|c|}{ NIVELES DE HUMO LÍQUIDO (\%) } & \multirow{2}{*}{$\mathrm{CV}$} \\
\hline & & 0,00 & & 0,30 & & 0,60 & & 0,90 & & \\
\hline \multirow{3}{*}{ Apariencia } & 10 & $4,75 \pm 0,25$ & a & $4,00 \pm 0,25$ & a & $4,25 \pm 0,25$ & a & $3,50 \pm 0,25$ & $\mathrm{~b}$ & 5,62 \\
\hline & 20 & $4,75 \pm 0,25$ & a & $4,00 \pm 0,25$ & a & $4,50 \pm 0,25$ & a & $3,50 \pm 0,25$ & $\mathrm{~b}$ & 1,43 \\
\hline & 30 & $3,50 \pm 0,25$ & a & $3,50 \pm 0,25$ & a & $3,25 \pm 0,25$ & a & $3,75 \pm 0,25$ & $\mathrm{a}$ & 9,42 \\
\hline \multirow{3}{*}{ Color } & 10 & $4,00 \pm 0,25$ & a & $4,00 \pm 0,25$ & a & $4,00 \pm 0,25$ & a & $3,50 \pm 0,25$ & a & 7,45 \\
\hline & 20 & $4,25 \pm 0,25$ & a & $4,00 \pm 0,25$ & a & $3,75 \pm 0,25$ & a & $3,75 \pm 0,25$ & a & 9,18 \\
\hline & 30 & $4,00 \pm 0,25$ & a & $4,00 \pm 0,25$ & a & $3,75 \pm 0,25$ & a & $3,75 \pm 0,25$ & a & 10,21 \\
\hline \multirow{3}{*}{ Sabor y Aroma } & 10 & $4,25 \pm 0,25$ & a & $4,50 \pm 0,25$ & a & $4,75 \pm 0,25$ & $\mathrm{a}$ & $4,00 \pm 0,25$ & $\mathrm{a}$ & 7,19 \\
\hline & 20 & $4,50 \pm 0,25$ & $\mathrm{a}$ & $4,75 \pm 0,25$ & a & $4,50 \pm 0,25$ & a & $4,00 \pm 0,25$ & $\mathrm{a}$ & 6,30 \\
\hline & 30 & $3,25 \pm 0,25$ & a & $3,50 \pm 0,25$ & a & $3,75 \pm 0,25$ & a & $3,50 \pm 0,25$ & a & 9,68 \\
\hline \multirow{3}{*}{ Textura } & 10 & $4,00 \pm 0,25$ & a & $4,00 \pm 0,25$ & a & $4,25 \pm 0,25$ & a & $3,25 \pm 0,25$ & a & 8,71 \\
\hline & 20 & $4,25 \pm 0,25$ & a & $4,25 \pm 0,25$ & $\mathrm{a}$ & $4,00 \pm 0,25$ & a & $3,50 \pm 0,25$ & a & 5,70 \\
\hline & 30 & $4,25 \pm 0,25$ & $\mathrm{a}$ & $4,00 \pm 0,25$ & $\mathrm{a}$ & $4,00 \pm 0,25$ & $\mathrm{a}$ & $3,50 \pm 0,25$ & $\mathrm{a}$ & 5,70 \\
\hline
\end{tabular}

Letras iguales indican diferencias no significativas $(p<0,05)$.

CV \%: Coeficiente de variación en porcentajes.

por el efecto de secado durante el almacenamiento; en los primeros días el producto mantiene una apariencia propia de un producto deseado, mientras que con el paso del tiempo, la tripa del producto, se va secando e incluso tiende a romperse, por lo que a la vista de los degustadores, este atributo pierde la característica esperada. Respecto al color los valores obtenidos de los distintos niveles de aplicación de humo líquido no difieren significativamente, esto se debe a que el producto (humo líquido) a pesar de que actúa sobre el color, las concentraciones utilizadas no inciden de manera significativa en el color, por lo que no ocurrió un cambio notorio de color. Sin embargo, al analizar más de cerca los datos de los tratamientos T0 y T1, se observó una mejor calificación, probablemente al color ligeramente más claro que tuvo mayor aceptación comparado con los tratamientos con mayor concentración de humo líquido (T2 y T3) que otorgó al chorizo un color levemente más oscuro.

En cuanto al aroma y sabor al inicio de la investigación, las muestras del chorizo ahumado en los distintos niveles de humo líquido presentaron valores con diferencia estadísticamente no significativo; esto puede deberse a que el humo líquido no actúa inmediatamente en el sabor. Se observó que humo líquido fue percibido en el chorizo de forma significativa con el aumento de concentración, la aceptación disminu- ye según la evaluación de los catadores. Este atributo mantuvo el grado de aceptación hasta el día 20, el cual empezó a caer, acercándose a valores mínimos de aceptación al día 30. Respecto a la textura al inicio de la investigación, el chorizo ahumado presentó valores que no difieren significativamente entre ellos; lo que puede deberse a que el humo líquido no es un insumo que determina la textura del producto. Sin embargo, pueden existir otros factores que determinen este atributo, siendo uno de ellos la misma tripa que se utilizó, lo cual le puede brindar una textura adecuada, ya que la tripa natural es muy fina. En estudios realizados en mariscos ahumados con humo líquido las características sensoriales se desarrollan de manera favorable a partir del día 20 hasta el día 30, también se determinó que los productos con mayor concentración de humo líquido presentaron colores más intensos, los cuales perdieron aceptación por los panelistas (Alcicek, 2012).

Los resultados del tiempo de vida útil son: 40, 41, 43 y 37 días que corresponden a T0, T1, T2 y T3 respectivamente. Las constantes cinéticas demuestran que T2 tuvo el menor valor, indicando el tiempo de vida útil más largo. Por otra parte, el tratamiento T3 tuvo la constante cinética más alta, indicando un menor tiempo de vida útil del producto. De esta manera se determinó que el T2 según las características organolépticas tiene mayor tiempo de vida útil (Figura 1). 


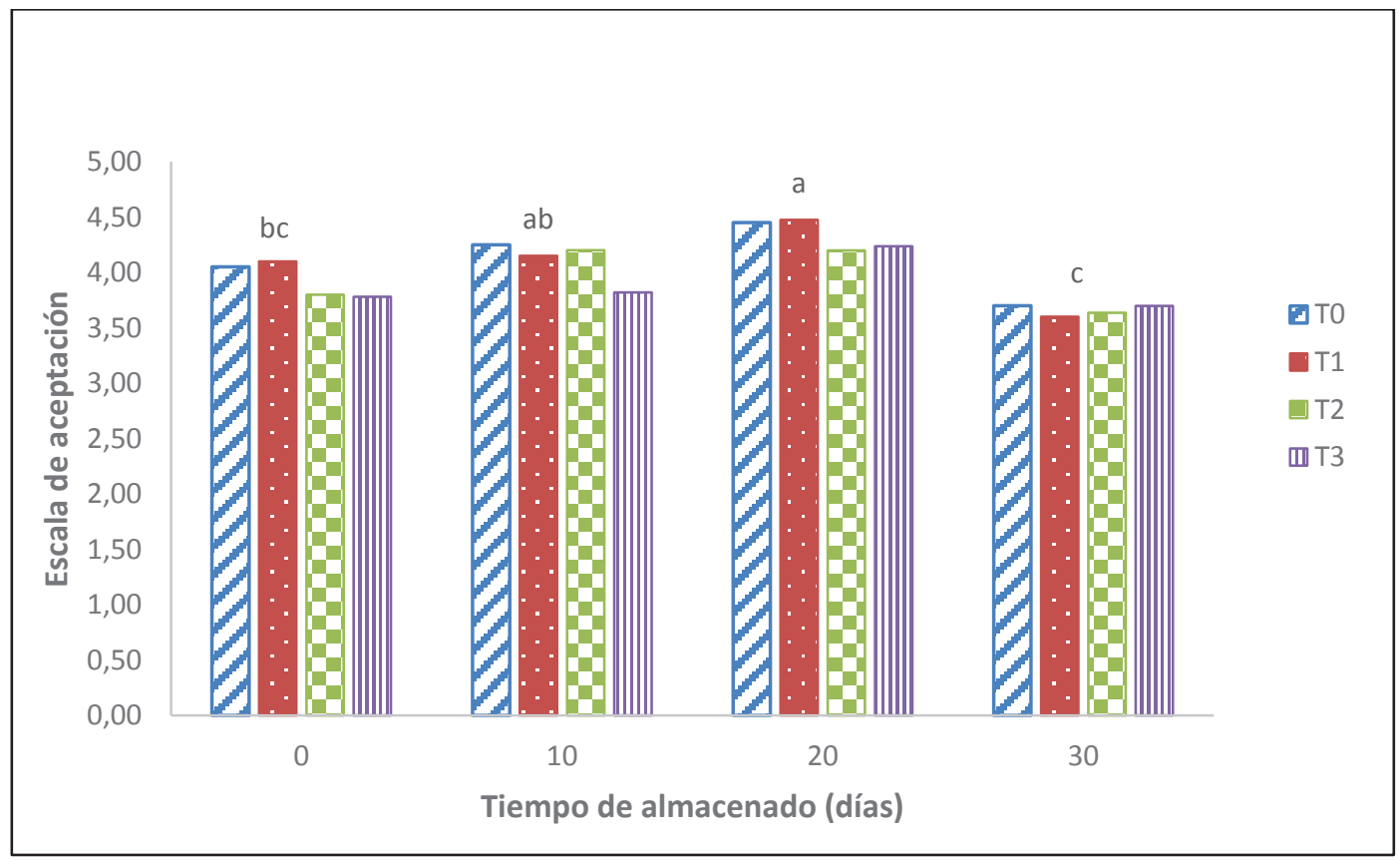

Figura 1. Comparación de la aceptabilidad del chorizo con diferentes concentraciones de humo líquido. Letras iguales indican diferencias no significativas $(\mathrm{p}<0,05)$.

Figure 1. Comparison of acceptability of sausage with different concentrations of liquid smoke. Similar letters indicate no significant differences $(\mathrm{p}<0.05)$.

\section{Características Microbiológicas}

Es evidente en el Cuadro 3 que, al comparar el tratamiento control T0 con los demás, siempre fue mayor la cantidad de microorganismos, lo que aumentó a medida que pasan los días de almacenado. Aunque en los demás tratamientos que contenían humo líquido hubo también crecimiento microbiano, éste fue siempre menor en relación al tratamiento control. La presencia de Escherichia coli en todos los tratamientos fue nula, por lo que se puede manifestar que el producto fue elaborado de forma higiénica respetando las normas de seguridad; sin embargo, de ello se puede decir que según las normas INEN 1338:2012 para carnes y productos cárnicos la presencia de Escherichia coli en el chorizo crudo y maduro es tolerable como máximo de 3,00 UFC/g. En relación a coliformes totales sí existió contaminación; a medida que avanzaron los días de almacenamiento la cantidad de coliformes totales aumentaron, pero ésta fue inversamente proporcional a la cantidad de humo líquido, es decir que a mayor cantidad, fue menor la concentración de coliformes totales. Del mismo modo, hasta el día 30 de almacenamiento el recuento de coliformes totales se mantuvo en cualquier nivel de humo líquido aplicado por debajo de los límites permitidos según la norma INEN 1338:2012. La presencia de hongos en el chorizo ahumado tuvo un comporta- miento similar al de coliformes totales, donde a mayor porcentaje de humo líquido, el crecimiento de microorganismos fue menor. Finalmente, hasta el día 30 el producto se mantuvo dentro de los rangos de tolerancia microbiológica, conforme la norma INEN 1338:2012 para carnes y productos cárnicos. Resultados similares se demuestran en estudios para determinar el tiempo de vida útil en embutidos, donde el humo líquido actuó como un inhibidor de crecimiento bacteriano (Özpolat y Patir, 2015). En otro tipo de productos como queso ahumado el comportamiento del humo líquido fue igual, evitó el crecimiento de bacterias lipolíticas, proteolíticas, hongos y levaduras, debido a las propiedades antimicrobianas del humo, específicamente por los compuestos fenólicos (Ammar et al., 2015).

Para la determinación de la vida útil del producto fue tomado únicamente los resultados de coliformes totales, ya que Escherichia coli no tuvo crecimiento en ninguna de las muestras, mientras que hongos se consideraron irrelevantes para el estudio de estabilidad del producto. Como se evidenció anteriormente, el tratamiento T0, es el que presentó un crecimiento mayor de coliformes totales en relación a los demás tratamientos. En la Figura 2 se observa este comportamiento en cada uno de los tratamientos y la línea de tendencia, la cual permitió calcular el tiempo de vida útil del producto, con los diferentes niveles de aplica- 
ción de humo líquido. Además, se puede observar que los valores de $\mathrm{R}^{2}$ para cada una de las curvas, son valores altos, por lo tanto, es posible correlacionar la estabilidad de la calidad microbiológica durante el almacenado como un modelo matemático. En el caso de los cuatro tratamientos, las líneas de tendencia se ajustan al modelo de cinética utilizado, por lo tanto, se realiza la regresión lineal, y se utiliza la fórmula de la recta de cada una de las líneas de tendencia para el cálculo del tiempo de vida útil. Finalmente, al aplicar la fórmula de cinética, se determinó que cuando la cantidad de coliformes totales llegue a $10 \mathrm{UFC} / \mathrm{g}$, el producto pierda la

Cuadro 3. Evolución de microorganismos en el chorizo con humo líquido durante el tiempo de almacenado.

Table 3. Microorganisms growth in sausage with liquid smoke during storage period.

\begin{tabular}{|c|c|c|c|c|c|c|}
\hline \multirow{2}{*}{ VARIABLES } & \multirow{2}{*}{$\begin{array}{l}\text { TIEMPO } \\
\text { (días) }\end{array}$} & \multicolumn{4}{|c|}{ NIVELES DE HUMO LÍQUIDO (\%) } & \multirow{2}{*}{ CV } \\
\hline & & 0,00 & 0,30 & 0,60 & 0,90 & \\
\hline \multirow{3}{*}{$\begin{array}{l}\text { Escherichia coli } \\
\text { (UFC/g) }\end{array}$} & 10 & 0,00 & 0,00 & 0,00 & 0,00 & \\
\hline & 20 & 0,00 & 0,00 & 0,00 & 0,00 & \\
\hline & 30 & 0,00 & 0,00 & 0,00 & 0,00 & \\
\hline \multirow{3}{*}{$\begin{array}{l}\text { Coliformes Totales } \\
\text { (UFC/g) }\end{array}$} & 10 & $5,00^{\text {a }}$ & $2,50^{\mathrm{a}}$ & $3,00^{\mathrm{a}}$ & $0,00^{\mathrm{a}}$ & 13,27 \\
\hline & 20 & $7,50^{\text {a }}$ & $5,50^{\mathrm{b}}$ & $5,00^{\mathrm{b}}$ & $2,50^{\mathrm{c}}$ & 9,85 \\
\hline & 30 & $9,00^{\text {a }}$ & $8,00^{\mathrm{a}}$ & $5,00^{\mathrm{a}}$ & $3,50^{\mathrm{c}}$ & 6,60 \\
\hline \multirow{3}{*}{ Hongos (UFC/g) } & 10 & $51,50^{\text {a }}$ & $59,00^{\text {a }}$ & $44,50^{\text {a }}$ & $20,00^{\text {a }}$ & 11,42 \\
\hline & 20 & $100,00^{a}$ & $110,00^{\mathrm{a}}$ & $87,50^{\text {a }}$ & $29,50^{\text {a }}$ & 7,98 \\
\hline & 30 & $225,00^{a}$ & $170,00^{\text {a }}$ & $109,75^{\text {a }}$ & $45,00^{\mathrm{a}}$ & 5,76 \\
\hline
\end{tabular}

Letras iguales indican diferencias no significativas $(\mathrm{p}<0,05)$.

CV \%: Coeficiente de variación en porcentajes.

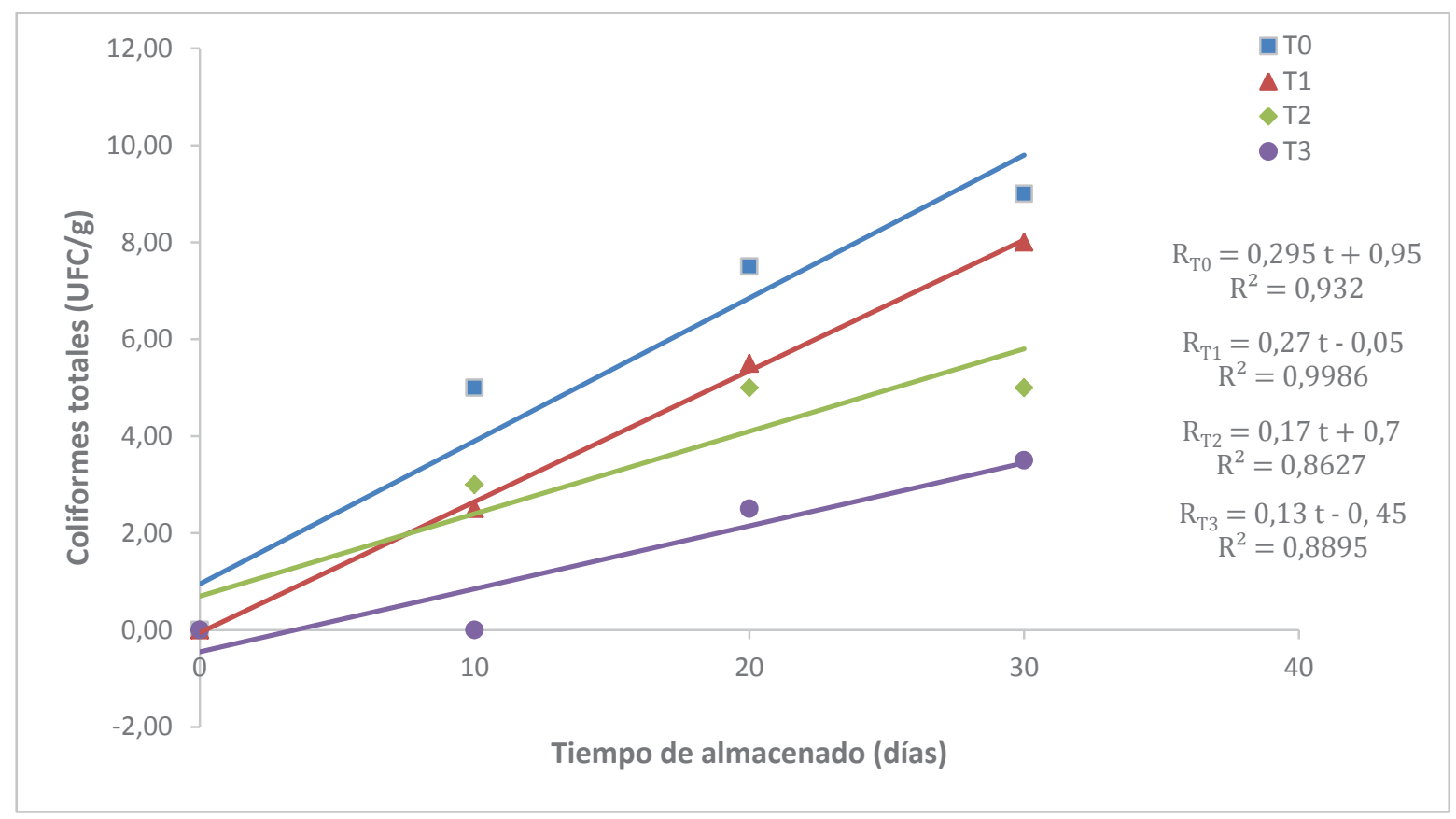

Figura 2. Recuento microbiológico de coliformes en el chorizo con diferentes concentraciones de humo líquido.

Figure 2. Microbiological count of coliformes in sausage with different concentrations of liquid smoke. 
inocuidad, por lo tanto, no es aceptable. En el Cuadro 4 se observa los diferentes valores de vida útil en relación a los parámetros microbiológico y organoléptico, y se observa que si bien el crecimiento microbiano del producto es lento y por lo tanto, prolonga la vida útil del mismo, la aceptabilidad decrece, es decir, la vida útil del producto lo determina el factor organoléptico. Finalmente, se determina que T2 tiene una vida útil más prolongada en relación a los demás tratamientos, siendo este valor de 43 días, lo cual se asemeja al periodo de vida útil de Salchicha Brasilera ahumada con humo líquido que es de 40 días (Schwert et al., 2011) y mayor en mariscos ahumados donde el tiempo de vida fue de 30 días (Alcicek, 2014). Además en comparación con otras marcas del mercado ecuatoriano de este tipo de chorizo (30 días), el tiempo de vida en esta investigación es mayor.

\section{CONCLUSIONES}

Microbiológicamente, el tratamiento T3 disminuye el desarrollo de Escherichia coli y coliformes totales y permite al producto tener un mayor tiempo de vida útil. A mayor concentración de humo líquido el crecimiento de microorganismos fue menor, con lo cual se puede concluir que el humo líquido tuvo un efecto preservante y antimicrobiano en el chorizo ahumado. Respecto a la aceptación del producto, existen 2 procesos, desde el día 0 hasta aproximadamente el día 20, las características sensoriales mejoraron con el pasar de los días; mientras que a partir de ese día la aceptación del mismo desciende, ya que comienza un pequeño cambio en el sabor del mismo, aunque hasta el día 30 de analizado el producto, todavía se mantuvo dentro de los rangos de aceptabilidad. Al comparar la cinética de crecimiento de los parámetros microbiológicos y organolépticos, se concluye que el tratamiento T2 procuró la vida útil más prolongada que fue de 43 días, dándole una ventaja frente a otros embutidos tipo chorizo que se encuentran en el mercado ecuatoriano, los cuales poseen una vida útil de 30 días, demostrando que el producto elaborado con humo líquido en el presente trabajo extiende la vida útil aproximadamente en casi dos semanas más en relación a productos actualmente en el mercado, lo cual es un punto atractivo para la industria cárnica.

\section{AGRADECIMIENTOS.}

Los autores agradecen el apoyo del Dr. Kong Shun Ah-Hen en la preparación y redacción del presente manuscrito para su publicación.

\section{REFERENCIAS}

Administración Nacional de Medicamentos, Alimentos y Tecnología Médica (ANMAT), 2006. Guía de Interpretación de resultados microbiológicos de alimentos. Instituto Nacional de Alimentos, Buenos Aires, Argentina,. http:// www.anmat.gov.ar/alimentos/Guia_de_interpretacion_ resultados_microbiologicos.pdf.

Alcicek, Z., 2012. Effects of Different Liquid Smoke Flavor Levels on the Shelf Life of Venus Clam (Chamelea gallina, L 1758) Meat. Journal of Food Processing and Preservation 38(3), 964-970. doi:10.1111/jfpp.

Ammar, E.-T., Ismail, M., El-Metwally, R., 2015. Effect of adding smoke liquid or powder to pickling whey on some pro-

Cuadro 4. Valores de constante cinético y tiempo de vida útil para los diferentes tratamientos.

Table 4. Values of kinetic constant and shelf-life for the different treatments.

\begin{tabular}{lccccc}
\hline Parámetros & T & $\mathbf{A}_{\mathbf{0}}$ & $\mathbf{k}$ & $\mathbf{A}$ & $\begin{array}{c}\text { V.U } \\
\text { (días) }\end{array}$ \\
\hline \multirow{3}{*}{ Coliformes Totales } & 0 & 0,475 & 0,295 & 10 & 65 \\
& 1 & 0,025 & 0,270 & 10 & 74 \\
Organoléptico & 2 & 0,350 & 0,175 & 10 & 114 \\
& 3 & 0,225 & 0,130 & 10 & 150 \\
\hline & 0 & 6,225 & 0,070 & 3 & 40 \\
& 1 & 5,325 & 0,056 & 3 & 41 \\
& 2 & 5,313 & 0,054 & 3 & 43 \\
\hline
\end{tabular}

$\mathrm{A}_{0}=$ valor inicial del factor al tiempo 0

$\mathrm{A}=$ valor límite del factor al tiempo $\mathrm{t}$

$\mathrm{k}=$ constante cinético

$\mathrm{T}=$ tratamiento 
perties of Domiatti cheese made from goat's milk. Carpathian Journal of Food Science and Technology 7(2), 124-136.

Amran, A.M., Abbas, A.A., 2011. Microbiological Changes and Determination of Some Chemical Characteristics for Local Yemeni Cheese. Jordan Journal of Biological Science 4(2), 93-100. http://jjbs.hu.edu.jo/files/v4n2/ Paper\%20No.5\%20modified\%20.pdf.

A.O.A.C., 1990. Association of Official Analytical Chemist. Official Methods of Analysis of the Association of Official Analytical Chemist. 15. ${ }^{\mathrm{a}}$ ed. Virginia.

Araya, E., 2003. Evaluación sensorial de los alimentos. Guía de Laboratorio Universidad de Chile. Facultad de Ciencias Agronómicas. Universidad de Chile, Santiago.

Barco, A., 2008. Embutidos, Procesamiento y Control de Calidad. $1^{a}$ edición. Editorial Ripalme, Perú. pp. 205 - 207

Barzola, S., 2013. Estudio de las cadenas pecuarias de Ecuador. Ministerio de Agricultura, Ganadería y Pesca de la Nación. Buenos Aires. Argentina. http://www.agroindustria.gob. ar/site/ganaderia/bovinos/05=Mercados/04=Carnes/_ archivos $/ 000002=$ Estudio $\% 20$ del $\% 20$ mercado $\% 20$ c\%C3\%A1rnico\%20de\%20Ecuador/000008-Estudio $\% 20$ del $\% 20$ mercado\%2 $\%$ c\%C3\%A1rnico\% 20 de\%20Ecuador.pdf

Cid, S., 2004. Humo líquido. Industria Alimenticia. http:// www.industriaalimenticia.com/articles/85223-humoliquido

Codex Alimentarius, 2015. Norma del Códex para la carne picada curada cocida. Codex Stan 98-1981 (Rev. 3.-2015).

Cordero, G., 2013. Aplicación del Análisis Sensorial de los Alimentos en la Cocina y en la Industria Alimentaria. Editorial Universidad Pablo de Olavide. Sevilla, España.

Essien, E., 2003. Fabricación de embutidos. Principios y práctica. Editorial Acribia. Zaragoza, España.

Gracey, J., 2001. Mataderos Industriales: Tecnología y Funcionamiento. Editorial Acribia, España.

Girard, J., 2000. Tecnología de la Carne y de los Productos Cárnicos. Editorial Acribia S.A., España.

Instituto Ecuatoriano de Normalización (INEN), 2012. Norma Técnica Ecuatoriana NTE INEN 1 338. Carne y productos cárnicos. Productos cárnicos crudos, productos cárnicos curados-madurados y productos cárnicos
Precocidos-cocidos. Requisitos. Primera Edición. Quito - Ecuador. http://www.normalizacion.gob.ec/wpcontent/uploads/downloads/2014/NORMAS_2014/ ACO/17122014/nte-inen-1338-3r.pdf

Jay, J., 2002. Microbiología Moderna de los Alimentos. $3^{\text {a }}$ edición. Editorial Acribia S.A. Zaragoza, España.

Milly, P., Toledo R., Ramakrishnan, S., 2005. Determination of minimum inhibitory concentrations of liquid smoke fractions. Journal of Food Science 70(1), 12-17.

Mira, J., 1998. Compendio de Ciencia y Tecnología de la Carne. Editorial Docucentro ESPOCH. Riobamba, Ecuador.

Moswittz, J., Howard, R., 1983. Products testing and Sensory evaluation of food. Editorial Inc. Wesport, Connecticut, Estados Unidos.

Ohloff, G., Flament, I., Pickenhagen, W., 1985. Flavor Chemistry. Food Reviews International 1(1), 99-148.

Özpolat, E., Patir B., 2015. Determination of shelf life for sausages produced from some freshwater fish using two different smoking methods. Journal of Food Safety. 36, 69-76. doi: 10.1111/jfs.12214.

Pico, P., 2008. Nociones Básicas de: Ahumado Artesanal y Profesional. www.byrd-multiequip.com.ar (acceso, 20.01.2009).

Ranken, M., 2003. Manual de Industrias de la Carne. Editorial Blackwell Science, Londres, Inglaterra.

Restrepo, C., 2012. Humo líquido naturalmente. Revista Alimentos. $29(6), 42-45$.

Sancho, J., 2002. Análisis Sensorial de los Alimentos. Editorial Alfa Omega. México.

Schwert, R., Verlindo, R., Cichoski, A.J., Oliveira, D., Valduga, E., 2011. Comparative evaluation of liquid and traditional smoke on oxidative stability, color and sensory properties of Brazilian calabrese sausage. CyTA - Journal of Food 9, 131-134. doi:10.1080/19476337.2010.491581.

Taoukis, P.S., Labuza, T.P., 1989. Applicability of Time-Temperature Indicators as Shelf Life Monitors of Food Products. Journal of Food Science 54(4), 783-788. doi: 10.1111/j.1365-2621.1989.tb07882.x.

Yusnaini, Y., Soeparno, I., Suryanto, E., Armunanto, R., 2012. Properties of Kenari Shell Liquid Smoke-immersedbeef. Journal of the Indonesian Tropical Animal Agriculture 37(1), 27-33. 
